\title{
Distancia interlingüística: partida de reflexiones metodológicas del español en el contexto chino
}

DOI: $10.32870 /$ mycp.v11i32.356

Lu Jingsheng*

\begin{abstract}
Spanish and Chinese are two very different languages on the linguistic forms level and on the cultural substance level. The learning of one presents several aspects and degrees of difficulty for the speakers of the other one. The analysis enables to assert that, like one of the resulting methodological reflections, it is very important to know the contrastive facets and always take them into account in the several learning and teaching aspects and processes of Spanish language for Chinese students.

Key words: interlinguistical distance, intercultural communication, methodology for Spanish as a foreign language, Spanish language for Chinese students.

Resumen

El español y el chino son dos idiomas muy diferentes tanto en las formas lingüísticas como en la sustancia cultural, y el aprendizaje del uno presenta diferentes aspectos y grados de dificultad para los hablantes del otro. El análisis permite afirmar que, como una de las consiguientes reflexiones metodológicas, es de importancia especial conocer las facetas de contraste y tenerlas siempre en cuenta en los diversos aspectos y procesos de la enseñanza y el aprendizaje del español para estudiantes chinos.

Palabras clave: distancia interlingüistica, comunicación intercultural, metodología de español como lengua extranjera, español para chinos.
\end{abstract}

Cuando estaba en Madrid en 1994 con una beca de estudio-investigación, asistí al Aula del Español que organizó la Universidad Antonio de Nebrija. Hubo una sesión de debate sobre ejercicios estructurales desarrollado entre dos profesores españoles especializados en la enseñanza del español como

* Director de la Facultad de Filologías Occidentales de la Universidad de Estudios Internacionales de Shanghai. 
segunda lengua. Ello me condujo a considerar que sí existen muchos esfuerzos por encontrar la metodología más adecuada o debates sobre si es positivo el ejercicio estructural en la enseñanza de lenguas de un estrecho parentesco, tales como español para hablantes de inglés, francés, italiano, hasta portugués, debe ser de importancia singular la búsqueda de una metodología adecuada y la insistencia en los ejercicios estructurales en la enseñanza del español a los hablantes chinos, ya que el español y el chino son dos idiomas muy diferentes tanto en la forma lingüística como en la sustancia cultural.

\section{Grados de diferencia intercultural}

En los estudios de la comunicación intercultural se plantea el concepto de grados de diferencia cultural entre comunidades culturales, la cual se puede representar sinópticamente en una cadena continua de mayor a menor:

Figura 1

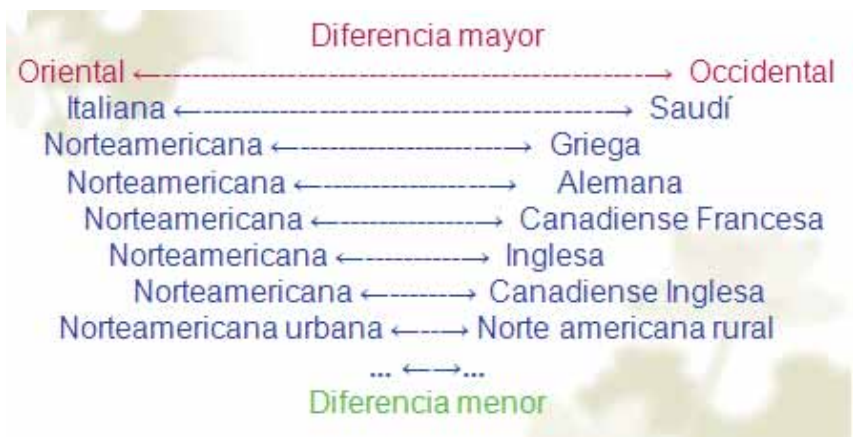

Fuente: Porter y Samovar, 1994.

La comunicación intercultural data de tiempo remoto, pero nunca como ahora es tan frecuente y rápida en esta aldea global. La reducción de la distancia temporal y espacial de comunicación en esta época contemporánea caracterizada por una sofisticada tecnología de comunicación, no ha logrado que desaparezca automáticamente la distancia sicológica, aun para aquellas personas de la misma comunidad lingüística. El idioma es sólo uno de los múltiples códigos utilizados en los contactos de la vida cotidiana y forma parte de la cultura como todo un sistema de signos. Las diferencias en cuanto a la orientación cultural, los valores, los conceptos, las normas sociales y los modos de ser, en mayor o 
menor grado entre diferentes comunidades lingüísticas y culturales conllevan otras diferencias en el proceso de la codificación y descodificación, en los actos lingüísticos y extralingüísticos, en las reglas pragmáticas, etcétera.

Cuando las personas se comunican en un idioma que, al menos para una de ellas, es extranjero, no es posible asegurar que los significados y valores compartidos con los que están imbuidos sean los mismos, cosa que se puede suponer cuando los hablantes se expresan en idiomas que comparten características similares (Byram y Fleming, 1998: 10).

Todo ello conduciría a problemas de comunicación entre gentes de diferentes lenguas y culturas, provocando una sensación de, según un dicho chino, estar separados en extremos del mundo aunque se encuentren cara a cara (zhi chi tian ya).

\section{Grados de distancia interlingüística}

En nuestras prácticas docentes de lenguas extranjeras creemos conveniente considerar también diferentes grados de la diferencia lingüística para aplicar adecuadamente la metodología.

Es obvio que no será igual, en términos cualitativos, la dificultad de un estudiante español para aprender francés que para aprender ruso, pues siendo el español y el francés dos lenguas pertenecientes a la misma familia lingüística, la distancia entre ambas será menor que la existente entre una lengua romance, el español, y una lengua eslava, el ruso (Santos Gallardo, 1994: 35).

\section{Figura 2}

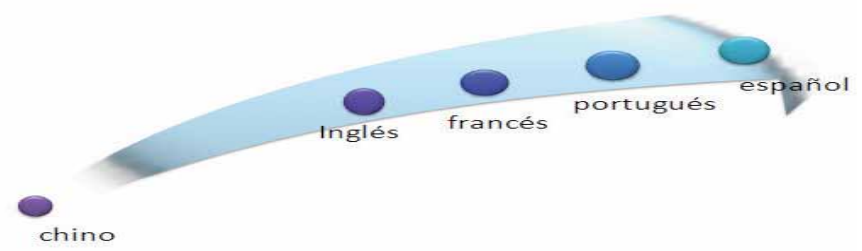

Fuente: elaboración propia.

En término general se puede decir que en la secuencia de distancia interlingüística, el chino como prototipo de las lenguas analíticas o aislantes y el español como ejemplo de las flexivas se encuentran en los dos extremos, entre ellos se 
halla el inglés y entre éste y el español se pueden situar las lenguas francesa, italiana y portuguesa, entre otras.

Tratamos de comprobar esta hipótesis con la siguiente tabla:

Tabla 1

Comparación lingüística entre chino, español, portugués e inglés

\begin{tabular}{|c|c|c|c|c|}
\hline & Chino & Inglés & Español & Portugués \\
\hline Genealogía & Chino-tibetana & \multicolumn{3}{|c|}{ Indo-europea } \\
\hline & & germana & \multicolumn{2}{|c|}{ latina } \\
\hline Escritura & Caracteres & & & \\
\hline Etimología & & $60 \%$ latín & $75 \%$ latín & \\
\hline Clase palabra & -art. +clasif. & \multicolumn{3}{|c|}{9 clases iguales } \\
\hline $\begin{array}{r}\text { Demarcación } \\
\text { de palabra }\end{array}$ & $\begin{array}{l}\text { Conceptual y } \\
\text { funcional }\end{array}$ & \multicolumn{3}{|c|}{ Formal (separabilidad) } \\
\hline Flexión & Casi nula & Algo & \multicolumn{2}{|c|}{ Abundante } \\
\hline Conversión & General & Mucha & \multicolumn{2}{|c|}{ Poca } \\
\hline Genero & - & - & \multirow{2}{*}{\multicolumn{2}{|c|}{$\begin{array}{l}\text { Sust. ar. adj.pron. } \\
\text { Sust. ar. adj.pron. }\end{array}$}} \\
\hline Número & - & Sust. & & \\
\hline $\begin{array}{r}\text { Conjugación } \\
\text { formas }\end{array}$ & - & -30 & $115-118$ & $119-123$ \\
\hline
\end{tabular}

Fuente: elaboración propia.

Genealógicamente, español, portugués, italiano, francés, etc. son como lenguas hermanas, y éstas y el inglés, alemán, etc. son como lenguas primas, y todas ellas no tienen ninguna relación familiar con el chino.

Según James I. Brown (2002), español e inglés tienen por lo menos más de $60 \%$ de su léxico que viene del mismo origen grecolatino, lo que no se conoce en absoluto en chino. Las lenguas europeas, en comparación, usan letras latinas como su escritura, que es totalmente diferente de la china pictográfica e ideográfica.

La clasificación de palabras en español e inglés sigue el mismo criterio clásico: nueve clases de palabra. En cuanto al chino, sin mencionar su complejidad en la clasificación, lo muy claro es que no tiene el artículo, pero sí un tipo de partículas llamadas medidor o clasificador $^{1}$ que no existe en las lenguas occidentales.

1. Partícula agregada a algunos nombres por convención, ej.: una ge persona, un pi caballo, un tou buey. 
Tanto el español y el portugués como el inglés tienen la palabra como unidad básica que lleva pausa virtual en el discurso y espacio divisorio en la escritura. Su delimitación es fácil debido a una de sus características más relevantes: la separabilidad (RAE, 1973: 163). La palabra española puede ser de un morfema, de varios morfemas, de una frase (ej.: cortaplumas) o de una oración compleja (ej.: correveidile), pero siempre se usa como un conjunto unido e inseparable.

En términos comparativos, es diferente la delimitación de la palabra entre los dos idiomas en cuestión. El chino destaca el criterio conceptual, pero el concepto es de por sí difícil de delimitar exactamente. Sin embargo, el criterio de la separabilidad tiene en cierto sentido un carácter absoluto en la delimitación de la palabra, por lo que facilita el conocimiento de la palabra en español.

El chino tiene la delimitación clara del morfema, mientras el español la tiene de la palabra. El español toma la separabilidad

El chino tiene la delimitación clara del morfema, mientras el español la tiene de la palabra como criterio para delimitar la palabra. Este procedimiento no produce dificultad para que el estudiante chino distinga las palabras en español. Sin embargo, debido a la trinidad de sílaba, morfema y carácter en su lengua materna, el estudiante chino está acostumbrado a conocer una palabra empezando por el morfema (carácter) para luego entender la palabra, lo que significa en la práctica un procedimiento de morfemas a palabras. La palabra española se presenta ante el estudiante chino como un bloque entero. En el aprendizaje del español se suele empezar por conocer la palabra para extenderse luego a la frase y a la oración.

En la morfología, español e inglés presentan similitudes en muchas categorías. Ambos tienen flexiones, pero en diferente grado: español tiene complicados accidentes en sus palabras: géneros, números, modos, tiempos y personas; un verbo de conjugación completa puede tener hasta un total de 115-118 formas flexivas, lo que constituye un verdadero rompecabezas para los chinos, habituados a su lengua básicamente aislante. El inglés no tiene cambios de género y el del número sólo en el sustantivo; su conjugación verbal, con unas 30 formas discutibles en criterio español, es mucho más sencilla que la española. 
Estos fenómenos gramaticales no suelen verse en chino. Las palabras conceptuales no llevan formas gramaticales, su categoría morfológica depende en gran medida de su distribución y función en el sintagma.

En la oración "Las clases son grandes y claras", cada una de las palabras tiene sus respectivos accidentes con los elementos gramaticales bien marcados:

Tabla 2

\begin{tabular}{cccc}
\hline Las & clases & son & grandes y claras \\
\hline artículo & sustantivo & verbo & adjetivo \\
pres./aux. & mas./fem. & modos & mas./fem. \\
det./indet. & sing./plur. & tiempos & sing./plur. \\
mas./fem. & forma de & personas & forma de plural \\
sing./plur. & $(-\mathrm{s} /$-es/ $\varnothing)$ & conj.reg./-irreg & $(-\mathrm{s} /$-es/ $\varnothing)$ \\
\hline
\end{tabular}

Fuente: elaboración propia.

Para cada palabra hay que hacer una selección en cada uno de los paradigmas expuestos y hay que atender al principio de correcta correlación (concordancia) entre las selecciones. Mientras tanto, la estructura de la versión china de la misma oración puede ser muy sencilla:

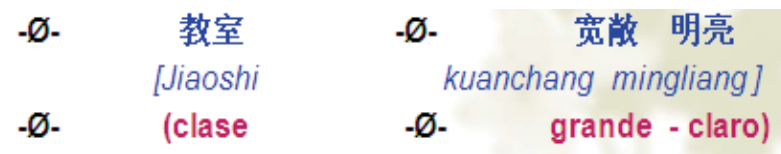

Fuente: elaboración propia.

La estructura sintáctica es parecida entre español e inglés, con orientación principal a la oración (sujeto-predicado), y muy distinta de la sintaxis china, orientada al discurso (tópico-comentario). Metafóricamente, la oración española es como una cadena de sintagmas con signo gramatical cuyas relaciones sintácticas son explícitas y formales, mientras la oración china puede considerarse una secuencia de conceptos cuyas relaciones sintácticas dependen, en gran dosis, del orden y el contexto en que se encuentran. El contraste de estructuras sintácticas se aprecia en la siguiente figura. 
Figura 4

Conceptos alineados
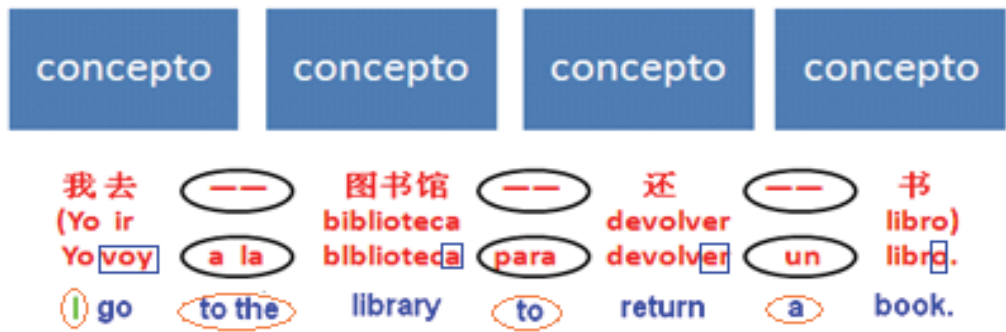

Español : conceptos encadenados con enlaces

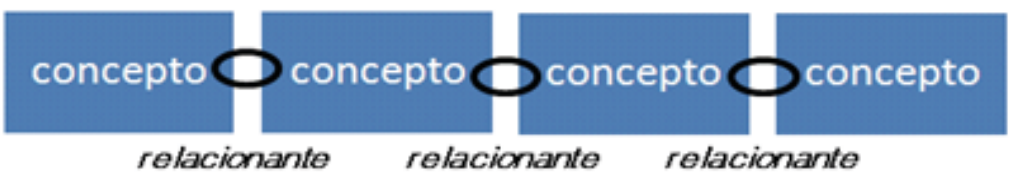

Fuente: elaboración propia.

Figura 5

Comparación de estructuras oracionales Esp: Elgobierno nos llamó a redoblar nuestros
esfuerzos por incrementar la producción.

Port. Ogoverno chamou-nos a redobrar os nossos esforços para incrementar a produçăo.

(con abundantes relacionantes)

Ing. The government called on us to redouble our efforts to increase production.

(con menos relacionantes que esbañol)

Chin. ...政府号召...我们...加倍努力...增加生产。

...gobierno llamar ...nosotros... redoblar esfuerzo

...aumentar producción

(sin relacionantes)

Fuente: elaboración propia. 
Comparativamente, la relación sintáctica española es rigurosa y complicada, la inglesa es menos complicada y la china es simplemente sencilla, como se ilustra en el siguiente grupo de ejemplos:

El sintagma chino tiene, en término general, el determinante delante del determinado, presentando una estructura cerrada que termina en el determinado. El español, mucho más libre en el orden de palabras, tiene una estructura sintagmática abierta que empieza a partir del determinado. Por estas características y debido a la capacidad limitada de memoria humana, la oración china, sobre todo en el lenguaje coloquial, suele ser mucho más corta que la española, que por sus elementos conjuntivos podría ser hasta infinitamente larga. Esto significa grandes dificultades en la traducción y la interpretación mutua, particular para una interpretación simultánea.

Por consiguiente los elementos gramaticales en la oración española tales como artículo, régimen prepositivo, pronombre, elementos conjuntivos, usos de modos y tiempos de verbo, estructura sintáctica de oraciones transitiva, intransitiva y pronominal en sus diferentes valores son complicados y constituyen diferentes grados de dificultad para los estudiantes chinos.

En cuanto a los aspectos semántico, estilístico y pragmático también existen problemas de comprensión y asimilación en el aprendizaje. Además, el español se habla en tantos países y tan distantes algunos de otros que se presentan muchas modalidades regionales con sus modismos propios, lo que significa otro problema para la enseñanza del español a alumnos chinos.

La cultura hispánica contiene una gran variedad de expresiones regionales y presenta ricas modalidades en diversas partes del mundo. No obstante, en término general se considera clasificada en el ámbito occidental, por lo que forma parte de la cultura occidental. La cultura china, milenaria y bastante tradicional, es típica del Oriente.

Obviamente son muy grandes las diferencias entre las lenguas china y española y entre las dos culturas, diferencias que existen en todos los niveles idiomáticos, pragmáticos y culturales. En muchos casos, por un descuido se provocarán problemas de comunicación, incluso en el plano fonético. Veamos un ejemplo. Tanto en español como en chino se registran seis fonemas oclusivos juntados en tres parejas. La diferencia consiste en que en español la oposición de cada pareja es entre sorda y sonora, mientras que en chino los seis fonemas oclusivos aparecen todos sordos, juntados en tres parejas aspirada-no aspirada. De este modo la distinción entre los fonemas sordos y sonoros no tiene mucha importancia funcional en chino, pero sí en español. 
Esto constituye una de las dificultades en el aprendizaje del español para los estudiantes chinos; no pocos suelen confundir los sonidos sonoros (b, $d, g$ ) por los sordos (p, t, k). Si se dijera (p) en lugar de (b) en el enunciado "Le invito a tomar una copa de vino", el error de pronunciación sería menor, quizá inadvertible sicológicamente, pues el contexto ayuda en la comprensión. Si se invitara a ver una función de la panda/banda china, el interlocutor lo sentiría un poco chistoso y preguntaría si tendría que ir al circo o al teatro. Si saludaran o se despidieran pidiendo un peso/beso, se provocaría molestia. Pero imagínese lo escandaloso que pudiera significar el mismo error cuando un guía de español explicara a los turistas antes de salir del hotel para la visita: "Ahora quiero llevarles a conocer un lugar donde hay una buda famosa y muchas budas bonitas".

\section{Reflexiones metodológicas}

Dada la gran diferencia entre el chino y el español, tenemos las siguientes reflexiones metodológicas en la enseñanza y aprendizaje de español en el contexto chino.

Es conveniente establecer un sistema didáctico basado en las características contrastivas de las dos lenguas y adecuado al aprendizaje de los alumnos chinos.

Consideramos muy importante el conocimiento de los aspectos contrastivos entre los dos idiomas para un buen profesor de español de los estudiantes chinos. En los departamentos de español de las universidades chinas trabajan fundamentalmente profesores chinos de español, quienes por sus experiencias de aprendizaje de español conocen los elementos comunes y distintos entre los dos idiomas y las dos culturas y por consiguiente saben el qué y el porqué de las dificultades de sus alumnos y el cómo orientarles a superar las mismas. Naturalmente, esta superioridad debe ser complementada por la presencia de profesores hispanohablantes nativos, a quienes se recomendaría hacer conocer características de la lengua y cultura china para que pongan en pleno juego sus superioridades en habilidades lingüística y cultural.

En la metodología didáctica, para los cursos de licenciatura se enfatiza un método integral perseguido con una distribución adecuada de asignaturas, combinando los modernos métodos audio-oral, estructural, comunicativo, nocio-funcional con los métodos tradicionales: de gramática y traducción. Para una asignatura determinada predomina alguno que otro método concreto 
y en el conjunto de asignaturas se juntan lo aprovechable y lo ventajoso de diversos métodos para conseguir el mayor rendimiento posible.

El uso de una lengua es prácticamente un hábito. Para usar las estructuras de nuestra lengua nativa, tenemos un conjunto de hábitos formados desde la niñez y por un largo proceso. Ahora nuestros alumnos — adultos ya - intentan dominar la lengua española en un nivel más alto posible, pero en un proceso más rápido posible y para ello tienen que conseguir el complejo sistema de nuevos hábitos que para ellos representa el uso del español. El único camino para llegar a la meta propuesta es hacer ejercicios intensivos y estructurales, muchas veces repetitivos e incluso monótonos, pero siempre con mucha fuerza, como propone el Instituto Cervantes en su nuevo Plan curricular:

Repetición mecánica: repetición reiterativa y mecánica del material susceptible de ser recordado. Ejemplo de aplicación: repetir, verbalmente o por escrito, listas de exponentes nocionales y funcionales, paradigmas de conjugación, listas de conectores, etc. de forma reiterada (Instituto Cervantes, 2006: 492).

Dadas las grandes diferencias que existen entre español y chino, sobre todo en el nivel cultural que es objeto de nuestra deliberación, es importante conocer diversas facetas culturales en contraste y fomentar la competencia intercultural del estudiante para formar el hablante intercultural (Byram y Fleming, 1998) capaz de establecer lazos entre su propia cultura y otras, de mediar y explicar la diferencia y, fundamentalmente, de aceptar esa diferencia y vislumbrar la humanidad subyacente que la compone. Hay que tener siempre en cuenta las diferencias culturales en los diversos aspectos de la enseñanza y el aprendizaje de las lenguas extranjeras, tales como la planificación curricular, la distribución de asignaturas, la metodología didáctica, la elaboración de materiales didácticos, la formación del docente, etcétera.

Es importante elaborar materiales didácticos de español adecuados al aprendizaje de los estudiantes chinos. Para unas asignaturas (sobre todo para tomos básicos de español, cursos de gramática, cursos de traducción e interpretación, etc.) hay que establecer un nuevo sistema cuyo contenido, forma y progreso se organizan según los objetivos concretos del curso y los problemas de aprendizaje de los estudiantes chinos. Para otras (conversación, lectura, audiovisual, etc.) se hacen adaptaciones a los materiales importados y a veces se usan éstos en su forma original. Hasta la fecha se han publicado varias decenas de libros, cintas, vídeos y VCD destinados a la enseñanza de español. 
Cabe mencionar la tenacidad de los hispanistas chinos para facilitar la comunicación entre los chinos y los hispanohablantes, reflejada en la elaboración de diccionarios bilingües entre los dos idiomas: más de 20 publicaciones entre las de bolsillo y las de volumen mayor que el DRAE.

Es importante diseñar bien el plan de estudios o la disposición de asignaturas. El plan de estudios lo determina cada centro docente. Aunque varía en algunos detalles, debe observar algunas normas generales. Se enfatiza una combinación de asignaturas prácticas y teóricas. Las prácticas se encaminan a que los estudiantes adquieran las cinco destrezas en el manejo del español, a saber: comprensión auditiva, expresión oral, lectura, expresión escrita y traducción; y las teóricas se imparten con el propósito de que los estudiantes profundicen en el conocimiento de la filología hispánica y de otros aspectos socioculturales del mundo hispánico.

\section{Referencias bibliográficas}

Brown, James I., Reading Power, Houghton Mifflin Company, Boston, 2002. Byram, M., y M. Fleming, Perspectivas intelectuales en el aprendizaje de idiomas (versión española), Cambridge University Press, Madrid, 2001 (1998). Fernández, Sonsoles, Interlengua y análisis de errores, Edelsa, Madrid, 1997. Instituto Cervantes, Enciclopedia del español en el mundo, Plaza y Janés, Barcelona, 2006.

- - Plan curricular del Instituto Cervantes. Niveles de referencia para el español, vols. 1-3, Edelsa, Madrid, 2007.

Lu, Jingsheng, Enseñanza e investigación del español en China, AAch, Madrid, 2000.

- - "Enseñanza del español en China. Las lenguas extranjeras en un mundo cambiante", Selección de textos del $11^{\circ}$ Encuentro Nacional de profesores de Lenguas Extranjeras, UNAM, México, 2005, pp. 67-84.

- - "Diseño de asignaturas por series y aplicación integral de métodos en la especialidad de lenguas extranjeras", Anthology of the first International Conference on Foreign Language Teaching Methodology in China, Waijiaoshe, Shangai, 2006, pp. 78-83.

Porter, R., y L. Samovar, An Introduction to Intercultural communication, Wadsworth Publishing Company, Belmont, California, 1994.

Real Academia Española, Esbozo de una nueva gramática de la lengua española, Espasa-Calpe, Madrid, 1973. 
- - Gramática descriptiva de la lengua española, dirigida por Ignacio Bosque y Violeta Demonte, 3 tomos, Espasa, Madrid, 1999.

Sánchez Lobato, J., e I. Santos Gargallo (dirs.), Vademécum para la formación de profesores. Enseñar español como segunda lengua (L2)/lengua extranjera (LE), SGEL, Madrid, 2005.

Santos Gallardo, I., Análisis contrastivo, análisis de errores e interlingüística en el marco de la lingüistica contrastiva, Síntesis, Madrid, 1994. 